\title{
CD73 as a novel marker for poor prognosis of oral squamous cell carcinoma
}

\author{
ZHEN-HU REN, YONG-XIANG YUAN, TONG JI and CHEN-PING ZHANG \\ Department of Oral and Maxillofacial-Head and Neck Oncology, Ninth People's Hospital, \\ Shanghai Jiao Tong University School of Medicine, Shanghai 200011, P.R. China
}

Received April 16, 2015; Accepted April 15, 2016

DOI: $10.3892 / \mathrm{ol} .2016 .4652$

\begin{abstract}
Ecto-5'-nucleotidase [cluster of differentiation (CD)73] has important functions in several types of cancer, however, its expression in squamous cell carcinoma (SCC) remains unknown. The present study was designed to investigate CD73 expression in SCC. CD73 expression was assessed by immunohistochemistry in 113 patients with oral SCC (OSCC). The association between CD73 expression and clinicopathological features, overall survival (OS) and disease-free survival (DFS) times of patients were statistically analyzed. CD73 expression was detected in $58.4 \%$ (66/113) of OSCC patients, with the immunostaining predominantly localized in the cytomembrane and a little in the cytoplasm. Statistical analysis revealed that CD73 expression was more frequently detected in patients with larger tumors $(\mathrm{P}=0.021)$. The overexpression of CD73 was significantly associated with clinical stage $(\mathrm{P}=0.047)$. Furthermore, immunohistochemical staining showed that overexpression of CD73 was inversely correlated with DFS $(\mathrm{P}=0.002)$ and $\mathrm{OS}(\mathrm{P}=0.002)$ times. Multivariate Cox regression analysis revealed that $\mathrm{CD} 73$ expression was an independent prognostic factor for poor DFS $(\mathrm{P}=0.018)$ and $\mathrm{OS}(\mathrm{P}=0.021)$. The current study is the first to evaluate the clinical significance and prognostic value of CD73 in patients with OSCC. The findings suggest that CD73 is a potential prognostic marker for OSCC.
\end{abstract}

\section{Introduction}

Despite improvements in treatment strategies for oral squamous cell carcinoma (OSCC), it remains one of the most devastating malignancies. OSCC is the sixth most common

Correspondence to: Dr Chen-Ping Zhang, Department of Oral and Maxillofacial-Head and Neck Oncology, Ninth People's Hospital, Shanghai Jiao Tong University School of Medicine, 639 Zhizaoju Road, Shanghai 200011, P.R. China

E-mail: zhangcp9th@hotmail.com

Key words: cluster of differentiation 73, oral squamous cell carcinoma, squamous cell carcinoma, prognosis, biomarker type of cancer in the world and accounts for almost $3 \%$ of all cancer cases (1-3). Although significant advancements have been made in the prevention, diagnostics and treatment strategies for OSCC, only modest progress has been made in improving the survival time in patients with progression or metastatic disease over the last 20 years $(4,5)$. In 2012, $\sim 300,400$ new cases of oral cavity cancer, including lip cancer, and $\sim 145,400$ associated mortalities occurred worldwide (6). Promising markers are the basis for improving the early detection and accurate survival evaluation of patients with OSCC.

Cluster of differentiation (CD)73, also known as 5'-nucleotidase (5'-NT) or ecto-5'-NT, is a 70-kD, glycosyl-phosphatidylinositol anchored cell surface enzyme that is encoded by the 5'-nucleotidase ecto gene. CD73 was originally defined as a lymphocyte differentiation antigen, and has both enzymatic and non-enzymatic functions (7). CD73 catalyzes the dephosphorylation of extracellular adenosine monophosphate to adenosine, promoting its suppressive effects on the immune system in the tumor microenvironment, invasion and metastasis of cancer (8). In addition to its enzymatic function, CD73 is also an adhesive and signaling molecule that mediates cancer invasion and metastasis (9). Thus, both the enzymatic and non-enzymatic functions of CD73 are involved in the processes of cancer occurrence and development.

Increased expression of CD73 expression has been observed in several types of malignancy $(10,11)$, such as breast cancer, prostate cancer, bladder cancer and malignant melanoma. In addition, it has prognostic value for patients with colon cancer and has been suggested as a diagnostic factor in papillary thyroid carcinoma (12). However, there is almost no information available regarding the survival influence of CD73 expression on tumor cells in patients with SCC. The current study analyzed the association between CD73 expression and clinicopathological characteristics, including disease-free survival (DFS) and overall survival (OS) time, in patients with OSCC.

\section{Materials and methods}

Enrolled patients. Patients with OSCC who underwent surgery (combined primary tumor resection and neck dissection/rescontruction) at the Department of Oral and Maxillofacial-Head and Neck Oncology, Ninth People's Hospital, Shanghai Jiao Tong University School of Medicine (Shanghai, China) were 
included in the present study. The inclusion criteria were as follows: i) Patients with OSCC (preoperative pathological diagnosis); ii) patients that underwent primary therapy in the Department of Oral and Maxillofacial-Head and Neck Oncology; iii) patients that did not receive preoperative chemotherapy, radiation therapy or any other treatment prior to surgery; and iv) patients diagnosed between January 2007 and December 2008. Cancerous and adjacent tumor samples were collected immediately after surgery. The distance between the tumor and the adjacent samples was $>2 \mathrm{~cm}$. According to the aforementioned criteria, 113 patients were included in the present study. The study was approved by the Ethics Committee of the Ninth People's Hospital, Shanghai Jiao Tong University School of Medicine. Informed consent was obtained from each patient.

Definition of $O S$ and DFS. OS time was calculated from the date of initial surgery to the date mortality from OSCC. If the patient did not succumb to OSCC, the end point was defined as mortality from any cause or the last date of follow-up. DFS was defined as the time from the date of initial surgery to the date of local or distant progression. If progression did not occur, the end point was defined as mortality from any cause or as the fifth year after surgery.

Immunohistochemical analysis. Sections $(4 \mu \mathrm{m})$ were cut from formalin-fixed (Mingsheng Disinfectant Co., Ltd., Chengdu, China) and paraffin-embedded (Shanghai Hualing Recovery Appliance Factory, Shanghai, China) tissues blocks of OSCC, and placed on positively charged glass slides (Citotest Labware Manufacturing Co., Ltd., Haimen, China). Following paraffin removal with xylene (Sangon Biotech Co., Ltd., Shanghai, China) and dehydration with ethanol absolute (Ling Feng Chemical Reagent Co., Ltd., Shanghai, China), the slides were steamed with $10 \mathrm{mmol} / \mathrm{l}$ citrate buffer (pH 6.0; DakoCytomation, Carpinteria, CA, USA) for $20 \mathrm{~min}$ for antigen retrieval. After cooling to room temperature, the sections were incubated overnight at $4^{\circ} \mathrm{C}(15 \mathrm{~h})$ with primary mouse monoclonal anti-CD73 antibody (1:50 dilution; cat no. sc-32299; Santa Cruz Biotechnology, Inc., Dallas, TX, USA). After several washes with phosphate-buffered saline, the sections were incubated with horseradish peroxidase-labeled goat anti-mouse or ant-rabbit secondary antibody (cat no. GK500705; Gene Tech, Shanghai, China) for $45 \mathrm{~min}$ at $37^{\circ} \mathrm{C}$, prior to adding diaminobenzidine (Dako, Glostrup, Denmark) for $3.5 \mathrm{~min}$ at room temperature.

Using an Axio Scope.A1 microscope (Carl Zeiss AG, Oberkocken, Germany), CD73 staining was independently evaluated by an expert pathologist who was blinded to the clinical information. The evaluation was analyzed according to the intensity of staining and the percentage of stained cells. Scoring estimations were stratified into four categories, as follows: +++, 20-49\% moderately to intensely stained cells or $\geq 50 \%$ positively stained cells;,$++ 10-19 \%$ intensively stained cells or $20-49 \%$ weakly stained cells;,$+ 10-19 \%$ weakly to moderately stained cells;,$\pm<10 \%$ positively stained cells; and -, $0 \%$ positively stained cells. For statistical analysis, + and above were recorded as positive; - and \pm were ranked as negative (13).

Statistical analysis. Comparisons of variables between the groups were based on the $\chi^{2}$ goodness-of-fit test or
Table I. Characteristics of the patients enrolled in this study $(\mathrm{n}=113)$.

\begin{tabular}{|c|c|}
\hline Characteristic & Value \\
\hline \multicolumn{2}{|l|}{ Age, years } \\
\hline Median (range) & $59.5(26-83)$ \\
\hline \multicolumn{2}{|l|}{ Gender, n (\%) } \\
\hline Male & $71(62.8)$ \\
\hline Female & $42(37.2)$ \\
\hline \multicolumn{2}{|l|}{ T stage, n (\%) } \\
\hline $\mathrm{T} 1$ & $44(38.9)$ \\
\hline $\mathrm{T} 2$ & $45(39.8)$ \\
\hline $\mathrm{T} 3$ & $15(13.3)$ \\
\hline T4 & $9(8.0)$ \\
\hline \multicolumn{2}{|c|}{ Clinical stage, n (\%) } \\
\hline I & $23(20.4)$ \\
\hline II & $23(20.4)$ \\
\hline III & $31(27.4)$ \\
\hline IV & $36(31.8)$ \\
\hline \multicolumn{2}{|c|}{ Differentiation, n (\%) } \\
\hline Well & $24(21.2)$ \\
\hline Moderate & $80(70.8)$ \\
\hline Poor & $9(8.0)$ \\
\hline \multicolumn{2}{|c|}{ CD73 cytology, n (\%) } \\
\hline Positive & $66(58.4)$ \\
\hline Negative & $47(41.6)$ \\
\hline \multicolumn{2}{|c|}{ Metastatic lymph nodes, n (\%) } \\
\hline Positive & $36(31.9)$ \\
\hline Negative & $77(68.1)$ \\
\hline
\end{tabular}

Fisher's exact test. DFS and OS times were estimated by the Kaplan-Meier life-table method. Univariate and multivariate parameters [gender, age, smoking, alcohol consumption, tumor stage (T stage) (14), lymph node metastasis, clinical stage (15), degree of differentiation] were analyzed with respect to DFS and OS using the Cox regression hazard model. Each experiment was repeated 3 times All analyses were performed using SPSS software (version 13; SPSS Inc., Chicago, IL, USA). $\mathrm{P}<0.05$ was considered to indicate a statistically significant difference.

\section{Results}

CD73 expression in primary OSCCs and its correlation with clinicopathological characteristics. The characteristics of enrolled patients are indicated in Table I. Positive staining was noted in 58.4\% (66/113) of OSCC samples. CD73 staining was predominantly localized in the cytomembrane with a small amount of staining in the cytoplasm. The OSCC samples showed marked overexpression of CD73 compared with matched adjacent noncancerous mucosa (Fig. 1). The association between CD73 expression and clinicopathological variables are indicated in Table II. Statistical analysis of the association between CD73 expression and clinicopathological 
Table II. Association between clinicopathological characteristics and CD73 expression in 113 patients with oral squamous cell carcinoma.

\begin{tabular}{|c|c|c|c|c|c|}
\hline \multirow[b]{2}{*}{ Clinicopathological feature } & \multirow[b]{2}{*}{ No. } & \multicolumn{2}{|c|}{ CD73 expression, $\%$} & \multirow[b]{2}{*}{$\chi^{2}$} & \multirow[b]{2}{*}{ P-value } \\
\hline & & Negative & Positive & & \\
\hline Gender & & & & 0.999 & 0.331 \\
\hline Male & 71 & 27 & 44 & & \\
\hline Female & 42 & 20 & 22 & & \\
\hline Age, years & & & & 2.004 & 0.184 \\
\hline$<60$ & 56 & 27 & 29 & & \\
\hline$\geq 60$ & 57 & 20 & 37 & & \\
\hline Smoking & & & & 0.065 & 0.844 \\
\hline Yes & 40 & 16 & 24 & & \\
\hline No & 73 & 31 & 42 & & \\
\hline Drinking & & & & 0.656 & 0.522 \\
\hline Yes & 31 & 11 & 20 & & \\
\hline No & 82 & 36 & 46 & & \\
\hline T stage & & & & 4.358 & 0.021 \\
\hline $\mathrm{T} 3 / \mathrm{T} 4$ & 24 & 5 & 19 & & \\
\hline $\mathrm{T} 1 / \mathrm{T} 2$ & 89 & 42 & 47 & & \\
\hline Lymph node metastasis & & & & 0.159 & 0.838 \\
\hline+ & 36 & 14 & 22 & & \\
\hline- & 77 & 33 & 44 & & \\
\hline Clinical stage & & & & 3.941 & 0.047 \\
\hline $\mathrm{III} / \mathrm{IV}$ & 46 & 14 & 32 & & \\
\hline $\mathrm{I} / \mathrm{II}$ & 67 & 33 & 34 & & \\
\hline Histological type & & & & 0.778 & 0.378 \\
\hline Poor & 9 & 5 & 4 & & \\
\hline Well/moderate & 104 & 42 & 62 & & \\
\hline
\end{tabular}

CD73, cluster of differentiation 73; T stage, tumor stage.

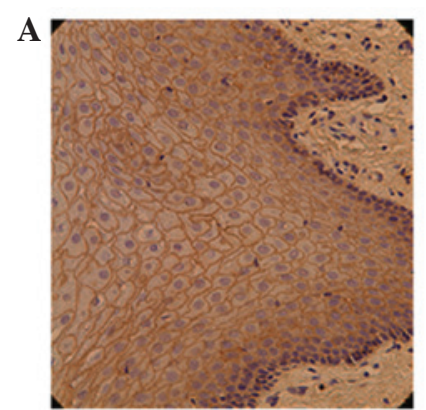

\section{D}

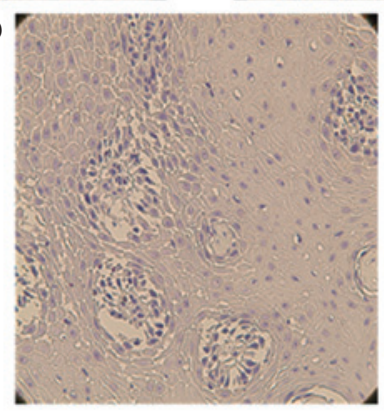

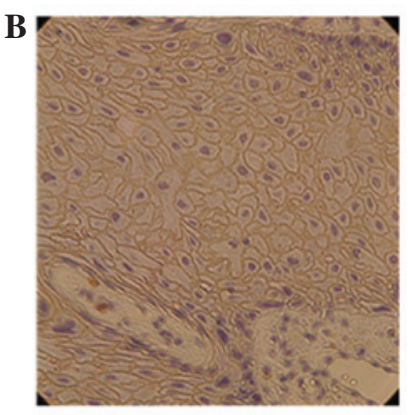
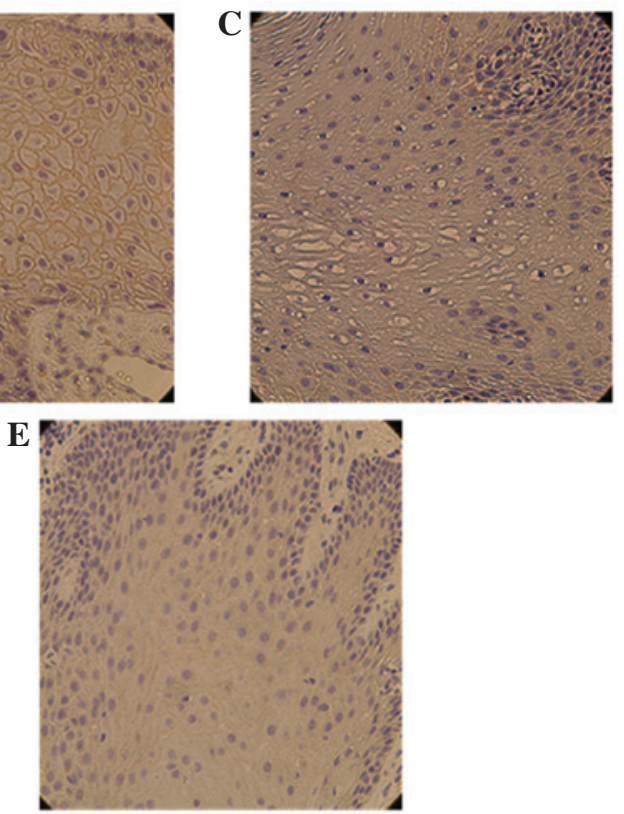

Figure 1. Association between CD73 expression in primary oral squamous cell carcinoma by immunohistochemical staining. (A),$+++($ B),$++($ C) + and (D) - scoring in OSCC. (E) - scoring in adjacent non-cancerous mucosa. Magnification, x400. 
Table III. Statistical analysis of clinicopathological variables associated with DFS and OS in patients with oral squamous cell carcinoma using the univariate Cox proportional hazards model.

\begin{tabular}{|c|c|c|c|c|c|c|}
\hline \multirow[b]{2}{*}{ Variable } & \multicolumn{3}{|c|}{ DFS } & \multicolumn{3}{|c|}{ OS } \\
\hline & HR & $95 \% \mathrm{CI}$ & P-value & HR & $95 \% \mathrm{CI}$ & P-value \\
\hline CD73 expression & & & 0.003 & & & 0.003 \\
\hline Positive vs. negative & 2.926 & $1.447-5.917$ & & 2.936 & $1.452-5.936$ & \\
\hline Gender & & & 0.584 & & & 0.567 \\
\hline Male vs. female & 1.189 & $0.640-2.210$ & & 1.199 & $0.645-2.228$ & \\
\hline Age, years & & & 0.060 & & & 0.057 \\
\hline$<60$ vs. $\geq 60$ & 1.027 & $0.999-1.055$ & & 1.027 & $0.999-1.056$ & \\
\hline Smoking & & & 0.751 & & & 0.751 \\
\hline Yes vs. no & 0.904 & $0.487-1.681$ & & 0.904 & $0.487-1.681$ & \\
\hline Drinking & & & 0.905 & & & 0.913 \\
\hline Yes vs. no & 0.961 & $0.496-1.860$ & & 0.964 & $0.498-1.866$ & \\
\hline T stage & & & 0.000 & & & 0.000 \\
\hline T3/T4 vs. T1/T2 & 1.797 & $1.342-2.408$ & & 1.816 & $1.356-2.432$ & \\
\hline Lymph node metastasis & & & 0.000 & & & 0.000 \\
\hline +vs. - & 1.897 & $1.344-2.687$ & & 1.915 & $1.358-2.701$ & \\
\hline Clinical stage & & & 0.000 & & & 0.000 \\
\hline III/IV vs. I/II & 1.677 & $1.282-2.194$ & & 1.698 & $1.298-2.220$ & \\
\hline Histological type & & & 0.008 & & & 0.009 \\
\hline Poor vs. well-moderate & 2.174 & $1.227-3.853$ & & 2.134 & $1.213-3.754$ & \\
\hline
\end{tabular}

DFS, disease-free survival; OS, overall survival; HR, hazard ratio; CI, confidence interval; CD73, cluster of differentiation 73; T stage, tumor stage.
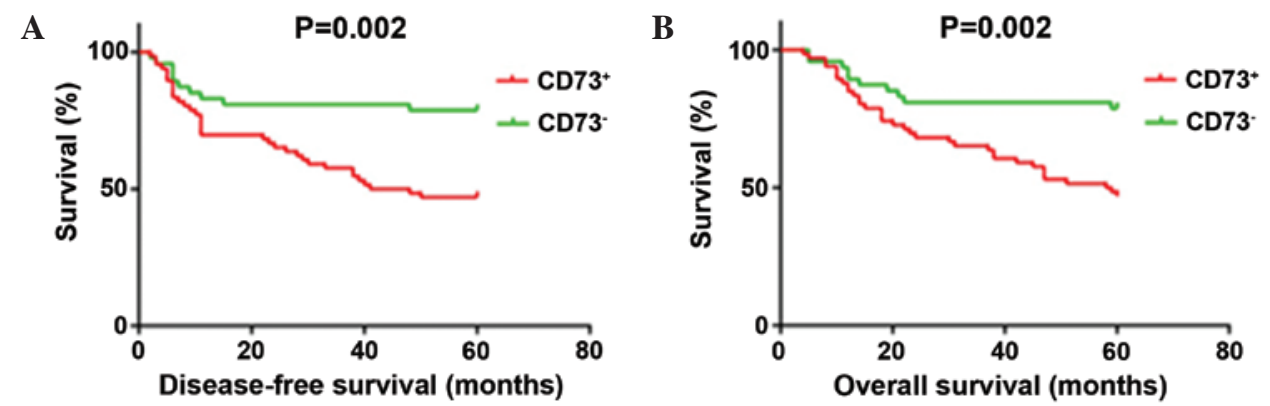

Figure 2. Survival analysis according to the expression status of CD73 for patients with oral squamous cell carcinoma. Kaplan-Meier survival curves for (A) disease-free survival and (B) overall survival in the study cohort ( $\mathrm{n}=113)$. CD73, cluster of differentiation 73.

characteristics identified that CD73 has a direct association with $\mathrm{T}$ stage $(\mathrm{P}=0.021)$ and clinical stage $(\mathrm{P}=0.047) . \mathrm{CD} 73$ expression was not significantly associated with other characteristics.

Association between CD73 expression, and DFS and OS times among patients with OSCC. Using follow-up data from the 113 patients with OSCC, the present study analyzed whether CD73 expression affected DFS and OS. Kaplan-Meier survival curves showed that CD73-positive expression cases had significantly poorer DFS $(\mathrm{P}=0.002)$ and $\mathrm{OS}(\mathrm{P}=0.002)$ times compared with CD73-negative cases. The 5-year DFS rate for positive CD73 expression was 47.0 compared with
$78.7 \%$ for patients that were negative for CD73; similarly, the 5-year OS rates for CD73-positive and -negative expression were 50.0 vs. $78.7 \%$, respectively (Fig. 2). After patients were stratified by $\mathrm{T}$ stage, cases with positive CD73 expression showed significantly poorer DFS and OS compared with CD73-negative cases among those classified as T1/T2 or clinical stage I/II. This trend was not observed in T3/T4 and clinical stage III/IV patients with OSCC (Fig. 3).

CD73 is an independent poor prognostic marker for DFS and OS times among patients with OSCC. Univariate Cox regression analysis showed that CD73 expression was significantly associated with poor DFS (HR, 2.926; 95\% CI, 1.447-5.917; 
Table IV. Statistical analysis of clinicopathological variables associated with DFS and OS in patients with oral squamous cell carcinoma using the multivariate Cox proportional hazards model.

\begin{tabular}{|c|c|c|c|c|c|c|}
\hline \multirow[b]{2}{*}{ Variable } & \multicolumn{2}{|c|}{ DFS } & \multirow[b]{2}{*}{ P-value } & \multicolumn{2}{|c|}{ OS } & \multirow[b]{2}{*}{ P-value } \\
\hline & HR & $95 \% \mathrm{CI}$ & & HR & $95 \% \mathrm{CI}$ & \\
\hline CD73 expression & & & 0.018 & & & 0.021 \\
\hline Positive vs. negative & 2.417 & $1.162-5.028$ & & 2.355 & $1.137-4.878$ & \\
\hline Gender & & & 0.947 & & & 0.897 \\
\hline Male vs. female & 1.025 & $0.486-2.165$ & & 1.050 & $0.498-2.214$ & \\
\hline Age, years & & & 0.087 & & & 0.070 \\
\hline$<60$ vs. $\geq 60$ & 1.028 & $0.996-1.061$ & & 1.030 & $0.998-1.063$ & \\
\hline Smoking & & & 0.757 & & & 0.715 \\
\hline Yes vs. no & 0.852 & $0.308-2.353$ & & 0.823 & $0.289-2.343$ & \\
\hline Drinking & & & 0.681 & & & 0.715 \\
\hline Yes vs. no & 0.795 & $0.267-2.369$ & & 0.810 & $0.262-2.505$ & \\
\hline T stage & & & 0.002 & & & 0.003 \\
\hline T3/T4 vs. T1/T2 & 2.735 & $1.428-5.236$ & & 2.598 & $1.369-4.932$ & \\
\hline Lymph node metastasis & & & 0.002 & & & 0.003 \\
\hline + vs. - & 2.775 & $1.443-5.337$ & & 2.633 & $1.376-5.039$ & \\
\hline Clinical stage & & & 0.040 & & & 0.060 \\
\hline I/II vs. III/IV & 0.464 & $0.223-0.964$ & & 0.500 & $0.243-1.030$ & \\
\hline Histological type & & & 0.025 & & & 0.045 \\
\hline Poor vs. well/moderate & 2.569 & $1.127-5.857$ & & 2.269 & $1.019-5.049$ & \\
\hline
\end{tabular}

DFS, disease-free survival; OS, overall survival; HR, hazard ratio; CI, confidence interval; CD73, cluster of differentiation 73; T stage, tumor stage.

$\mathrm{P}=0.003)$ and $\mathrm{OS}(\mathrm{HR}, 2.936 ; 95 \% \mathrm{CI}, 1.452-5.936 ; \mathrm{P}=0.003)$ times of patients with OSCC (Table III). Multivariate analysis using the Cox regression hazard model confirmed that CD73 expression was an independent prognostic factor for poor DFS (HR, 2.417; 95\% CI, 1.162-5.028; P=0.018) and OS (HR, 2.355; 95\% CI, 1.137-4.878; P=0.021; Table IV) among patients with OSCC. Multivariate analysis also indicated that T stage, clinical stage, degree of differentiation and lymph node metastasis have independent prognostic value in OSCC (Table IV).

\section{Discussion}

To the best of our knowledge, the current study is the first to evaluate the expression status of CD73 in patients with OSCC. The associations between CD73 expression and clinicopathological characteristics were evaluated in patients with OSCC. The findings indicated that positive CD73 expression may be a novel prognosticator of adverse clinical outcome in patients with OSCC. In addition, the present results identified that CD73 expression status was statistically associated with the $\mathrm{T}$ stage, clinical stage, degree of differentiation and lymph node metastasis. According to these findings, we propose that $\mathrm{CD} 73$ may be a novel molecular prognostic marker in the evaluation of OSCC patient survival. The current findings may be the beginning of a new era of research into the role of CD73 in SCC.

Overexpression of $\mathrm{CD} 73$ has been observed in broad types of cancer (16) and a growing body of literature has revealed the function of CD73 in cancer progression. For example, overexpression of CD73 was significantly associated with a worse prognosis in patients with triple negative breast cancer (17). By contrast, a number of retrospective studies reported that overexpression of CD73 was strongly correlated with improved clinical outcome in patients with breast cancer $(18,19)$. Furthermore, Lu et al (20) investigated the expression status of CD73 in gastric cancer, and showed that CD73 expression was positively associated with cancer stage, depth of invasion and metastasis, with low OS observed in the patients with overexpression of CD73. In addition, numerous studies have revealed that high levels of CD73 are statistically associated with a poor prognosis in colorectal cancer and gallbladder cancer $(21,22)$. Zhao et al (23) reported the CD73 status in various leukemia subtypes, and found the CD73 status was correlated with leukemia subtype and differentiation. In addition, a retrospective study reported that CD73 overexpression was positively correlated with lymph node metastasis in prostate cancer, and was more frequently observed in epithelial ovarian cancer patients with better prognosis, lower stage and better differentiation $(19,24)$. Similar results were observed in malignant melanoma (25). Taken together, the aforementioned findings indicate that CD73 is a significant molecular prognosticator in various types of cancer. However, there is almost no information regarding CD73 expression in the tumor cells of patients with SCC. Therefore, the current study aimed to conduct research in this field. 

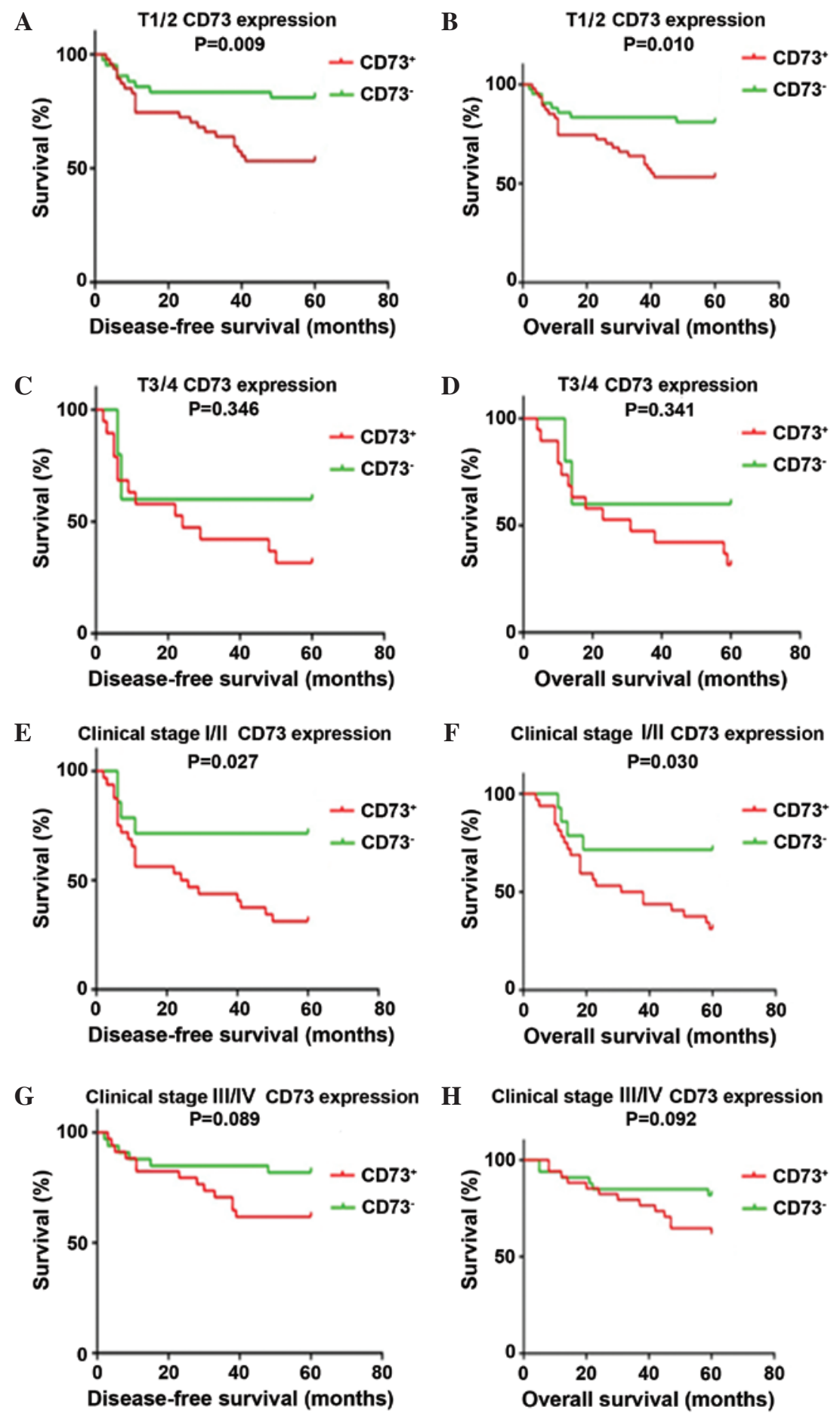

Figure 3. Kaplan-Meier survival curves showing disease-free survival (DFS) and overall survival (OS) in patients with oral squamous cell carcinoma (OSCC) according to T stage or clinical stage. CD73 expression is associated with (A) DFS or (B) OS among OSCC patients with T1/T2 tumors but is not significantly associated with (C) DFS and (D) OS among patients with T3/T4 tumors. CD73 expression is not associated with (E) DFS or (F) OS among OSCC patients in clinical stage I-II but is not significantly associated with DFS (G) and OS (H) among patients in clinical stage III-IV. T stage, tumor stage; CD73, cluster of differentiation 73 .

T stage and clinical stage are the most important prognostic markers in the evaluation of OSCC patient survival $(26,27)$. The current findings confirmed the validity of the $\mathrm{T}$ stage and clinical stage at the molecular level. Furthermore, CD73 may be a crucial molecular prognostic marker in early $\mathrm{T}$ stage (T1/T2) or early clinical stage (I/II) OSCC.

The limitations of the present study include the retrospective nature of the study and the limited number of enrolled patients. The findings of the study should be replicated in a randomized prospective study that includes a large sample size. Additional molecular studies are required to confirm whether CD73 has an important role in OSCC progression. The current authors are currently performing a further molecular study to confirm whether CD73 has an important role in OSCC.

In conclusion, the results of the present study revealed upregulation of CD73 in clinical OSCC tissues. In addition, it 
was identified that the grading of CD73 expression by immunohistochemical staining was able to significantly predict OSCC prognosis in a multivariate analysis. Furthermore, the CD73 expression status was significantly associated with $\mathrm{T}$ stage and clinical stage, and overexpression of CD73 was inversely correlated with the DFS and OS times of patients with OSCC. Thus, the results of the present study consistently suggest that CD73 is likely to be a novel prognostic marker of OSCC and holds promise for future tailored treatment strategies.

\section{References}

1. Jemal A, Bray F, Center MM, Ferlay J, Ward E and Forman D: Global cancer statistics. CA Cancer J Clin 61: 69-90, 2011.

2. Warnakulasuriya S: Global epidemiology of oral and oropharyngeal cancer. Oral Oncol 45: 309-316, 2009.

3. Ren $\mathrm{Z}$ and $\mathrm{Wu} \mathrm{H}$ : Advances in radical surgery of tongue cancer. J Prac Stomatology 30: 110-114, 2014 (In Chinese).

4. Silverman S Jr: Demographics and occurrence of oral and pharyngeal cancers. The outcomes, the trends, the challenge. J Am Dent Assoc 132 (Suppl): S7-S11, 2001.

5. Chandler K, Vance C, Budnick S and Muller S: Muscle invasion in oral tongue squamous cell carcinoma as predictor of nodal status and local recurrence: Just as effective as depth of invasion? Head Neck Pathol 5: 359-363, 2011.

6. Torre LA, Bray F, Siegel RL, Ferlay J, Lortet-Tieulent J and Jemal A: Global cancer statistics, 2012. CA Cancer J Clin 65 : 87-108, 2015.

7. Stagg J and Smyth MJ: Extracellular adenosine triphosphate and adenosine in cancer. Oncogene 29: 5346-5358, 2010.

8. Ghiringhelli F, Bruchard M, Chalmin F and Rébé C: Production of adenosine by ectonucleotidases: A key factor in tumor immunoescape. J Biomed Biotechnol 2012: 473712, 2012.

9. Zhi X, Chen S, Zhou P, Shao Z, Wang L, Ou Z and Yin L: RNA interference of ecto-5'-nucleotidase (CD73) inhibits human breast cancer cell growth and invasion. Clin Exp Metastasis 24 439-448, 2007.

10. Wang L, Tang S, Wang Y, Xu S, Yu J, Zhi X, Ou Z, Yang J, Zhou P and Shao Z: Ecto-5'-nucleotidase (CD73) promotes tumor angiogenesis. Clin Exp Metastasis 30: 671-680, 2013.

11. Arozarena I, Sanchez-Laorden B, Packer L, Hidalgo-Carcedo C, Hayward R, Viros A, Sahai E and Marais R: Oncogenic BRAF induces melanoma cell invasion by downregulating the cGMP-specific phosphodiesterase PDE5A. Cancer Cell 19: 45-57, 2011.

12. Zhang B: CD73 promotes tumor growth and metastasis. Oncoimmunology 1: 67-70, 2012.

13. Xing XF, Li H, Zhong XY, Zhang LH, Wang XH, Liu YQ, Jia SQ, Shi T, Niu ZJ, Peng Y, et al: Phospholipase A2 group IIA expression correlates with prolonged survival in gastric cancer. Histopathology 59: 198-206, 2011.
14. Patel SG and Shah JP: TNM staging of cancers of the head and neck: striving for uniformity among diversity. CA Cancer J Clin 55: 242-258; quiz 261-262, 264, 2005.

15. Greene FL and Sobin LH: The staging of cancer: A retrospective and prospective appraisal. CA Cancer J Clin 58: 180-190, 2008.

16. Gao ZW, Dong K and Zhang HZ: The roles of CD73 in cancer. Biomed Res Int 2014: 460654, 2014.

17. Loi S, Pommey S, Haibe-Kains B, Beavis PA, Darcy PK, Smyth MJ and Stagg J: CD73 promotes anthracycline resistance and poor prognosis in triple negative breast cancer. Proc Natl Acad Sci USA 110: 11091-11096, 2013.

18. Supernat A, Markiewicz A, Welnicka-Jaskiewicz M, Seroczynska B, Skokowski J, Sejda A, Szade J, Czapiewski P, Biernat W and Zaczek A: CD73 expression as a potential marker of good prognosis in breast carcinoma. Appl Immunohistochem Mol Morphol 20: 103-107, 2012.

19. Oh HK, Sin JI, Choi J, Park SH, Lee TS and Choi YS: Overexpression of CD73 in epithelial ovarian carcinoma is associated with better prognosis, lower stage, better differentiation and lower regulatory $\mathrm{T}$ cell infiltration. J Gynecol Oncol 23: 274-281, 2012.

20. Lu XX, Chen YT, Feng B, Mao XB, Yu B and Chu XY: Expression and clinical significance of CD73 and hypoxia-inducible factor-1 $\alpha$ in gastric carcinoma. World J Gastroenterol 19: 1912-1918, 2013.

21. Wu XR, He XS, Chen YF, Yuan RX, Zeng Y, Lian L, Zou YF, Lan N, Wu XJ and Lan P: High expression of CD73 as a poor prognostic biomarker in human colorectal cancer. J Surg Oncol 106: 130-137, 2012.

22. Xiong L, Wen Y, Miao X and Yang Z: NT5E and FcGBP as key regulators of TGF-1-induced epithelial-mesenchymal transition (EMT) are associated with tumor progression and survival of patients with gallbladder cancer. Cell Tissue Res 355: 365-374, 2014.

23. Zhao SX, Zhang HM, Dong SX, Liu JH, Zhou Z, Wang HJ, Zhu XF, Mi YC and Ru YX: Characteristics and clinical significance of CD73 expression in subtypes of leukemia. Zhongguo Shi Yan Xue Ye Xue Za Zhi 19: 1141-1144, 2011 (In Chinese).

24. Yang Q, Du J and Zu L: Overexpression of CD73 in prostate cancer is associated with lymph node metastasis. Pathol Oncol Res 19: 811-814, 2013

25. Wang $\mathrm{H}$, Lee $\mathrm{S}$, Nigro $\mathrm{CL}$, Lattanzio L, Merlano $\mathrm{M}$, Monteverde M, Matin R, Purdie K, Mladkova N, Bergamaschi D, et al: NT5E (CD73) is epigenetically regulated in malignant melanoma and associated with metastatic site specificity. Br J Cancer 106: 1446-1452, 2012.

26. Jardim JF, Francisco AL, Gondak R, Damascena A and Kowalski LP: Prognostic impact of perineural invasion and lymphovascular invasion in advanced stage oral squamous cell carcinoma. Int J Oral Maxillofac Surg 44: 23-28, 2015.

27. Lawaetz M and Homøe P: Risk factors for and consequences of inadequate surgical margins in oral squamous cell carcinoma. Oral Surg Oral Med Oral Pathol Oral Radiol 118: 642-646, 2014. 